\title{
Conjectures and Disconfirmations: Confirming the Standard Model Higgs
}

\section{Martin King ${ }^{1}$}

Received: 23 July 2020 / Accepted: 21 September 2021

(c) The Author(s) 2021

\begin{abstract}
One model in particular, the Standard Model Higgs, is taken to have been confirmed by the Higgs boson discovery at the LHC, even though many models are compatible with the data. Some models even provided riskier predictions and should perhaps be regarded as having been even more strongly confirmed. This paper sketches an argument demonstrating this by comparing the confirmation of the Standard Model Higgs with that of the Higgs in minimal supersymmetry. The paper then attempts to provide a way of understanding this result by modelling it as a case of eliminative induction.
\end{abstract}

\section{Introduction}

New scientific models are constantly developed to uncover and explain the unknown and to better explain the known. They are often designed by construction to accommodate existing data and though they may disagree significantly from each other in ontology and concept, they may only disagree about the values of observables by amounts that are currently indistinguishable. There are so many models with overlapping predictions, that determining which of these models and hypotheses are supported by results of new empirical evidence and which are unaffected or undermined is a very complex and nuanced affair. A central aim of the paper is to uncover some of the complexities of confirmation in a contemporary setting by taking a detailed look at a scientific discovery and the reasoning behind claims of confirmation. I propose that this case and cases under similar conditions can be understood as a kind of eliminative induction, where the confirmation of a model comes largely from the disconfirmation of some of its many alternative models.

The case study of interest is the discovery of a Higgs boson at the Large Hadron Collider (LHC) in 2012. I examine the effect of the discovery on the predictions of the dominant theory of particle physics, the Standard Model (SM), in comparison

Martin King

mking@uni-bonn.de

1 Physics Institute, University of Bonn, Bonn, Germany 
to that of a popular model of beyond the Standard Model (BSM) physics, minimal supersymmetry. Prior to the discovery, many models had been proposed that predicted some new particle, called a Higgs boson, would be found that is indicative of a mechanism responsible for generating the masses of the fundamental particles. Even though many of the allowable mass ranges and predicted properties of these Higgs particles strongly overlap with discovered particle, the discovery is touted as a victory of one model of the Higgs in particular, namely, the Standard Model Higgs. A Higgs boson was certainly discovered, but since the discovery, many particle physicists have focused on determining what kind of Higgs it is. Analysing this requires taking a step beyond the confirmation of $a$ Higgs, or of the Higgs mechanism [as undertaken in Dawid (2017)], and examining the reasoning that distinguishes between different Higgs models.

\section{Scientific Confirmation}

We have trustworthy and successful empirical generalisations, but justifying knowledge of universal statements is notoriously problematic. Confirmation does not concern justification of certain knowledge, as was the project of many modern philosophers, but rather degrees of confidence. The degrees of confidence can be gauged by comparing the empirical consequences of a scientific model with data. ${ }^{1}$ These degrees of confidence can also be conveniently spelled out in terms of probabilities and a theory of confirmation can indicate how one's probability assignments should change as new data is taken into account.

Confirmation theory was originally developed in a context where scientific theories were understood as axiomatic systems in a formal language (e.g. Hempel, 1965). Here, one treated the confirmation of hypotheses in terms of propositions about observables deduced from the theorems or axioms of a theory. In a more contemporary setting, one can think of theories as collections of models or simply forego talk of theories and focus on the confirmation of models. Scientific models are not linguistically formulated theorems of an axiomatic system and do not deductively entail propositions that can be interpreted as predictions about observations that can be determined by simple hypothesis testing. A better way to picture it is that models 'entail' some observations in the sense that it has been calculated with a certain degree of confidence that certain values of measurable parameters will fall within a specific range given the empirically or theoretically determined values of other parameters. In contemporary confirmation assessments, one also has to deal with the fact that there are many models that can accommodate known data as precisely as it has been determined. These models are not empirically equivalent, as their empirical consequences can be distinguished, but they are empirically adequate, and exhibit a kind of underdetermination that has been called transient. They can accommodate current data, but could be distinguished by future data. I will often refer to these models as 'viable' to stress that they are still on the table for

\footnotetext{
1 Though some have argued for the possibility of non-empirical confirmation, such as (Dawid et al., 2015).
} 
particle physicists. Because models can have highly-adjustable parameters and allow for broad, overlapping ranges of parameter values, determining in which models we should have increased or decreased confidence is not straightforward. Making these determinations is an essential aim of science and the complexity involved marks the central problematic of this paper.

As Carnap pointed out, one should be careful to distinguish between two distinct concepts of confirmation (Carnap, 1962). Confirmation can be thought of in an absolute sense, where it is an assessment of confidence in a theory, or in a relative sense, where it is a measure of the change in confidence before and after some evidence. Carnap calls the first "confirmation as firmness" and the second "confirmation as increase in firmness". ${ }^{2}$ I will avoid confusing these by referring to confirmation only in the second sense and emphasising the confirmation by an experiment; when I speak of firmness, I will speak of confidence or support.

In order to see some of the difficulties, let us briefly consider a well-known and often discussed case of confirmation, the Eddington expedition that measured the bending of light around the Sun during an eclipse. ${ }^{3}$ This famous experiment is widely regarded as having demonstrated Einstein's victory over Newton. The idea of the experiment was to test whether and to what degree light is deflected when passing close to the Sun. Einstein's theory predicted that the light from distant stars would be deflected by $1.75 \mathrm{~s}$ of arc when passing directly next to the Sun. On the other hand, Einstein could only be rebuffed if it was found that the amount of deflection was $0.87 \mathrm{~s}$ of arc, or if there was none at all. Through the analysis of two distinct measurements, it was determined (not uncontroversially) that there was a deflection of light and that it agreed much more strongly with the GR prediction than the Newtonian. There were many who suggested alternative explanations, but these were all eventually found wanting, in particular because they would entail other deviations that would have been observed in other experimental contexts. The alternative theories that up until that point had been empirically equivalent, could now be distinguished and the reasonable course of action was to accept that GR had been confirmed and its rivals disonconfirmed by the experiment.

By contrast, the Higgs case study (Sect. 3) is comparatively more complex. If the Eddington expedition were more analogous to the Higgs case study, it would be as though there were a huge number of theories of gravity, many of which predicted the deflection of light, and some by only observationally indistinguishable amounts. Imagine that some models could be used to predict only very wide ranges, say $0.5-2 \mathrm{~s}$ of arc, others a smaller range, say 1.5-2 s of arc, and one that only predicts that there would be some deflection but could provide no quantitative prediction. All of these models would have been compatible with the data from the Eddington expedition. Would they all have been confirmed the same amount or were some confirmed more than others? Does the range of allowed values matter? Does this tell you anything about gravity if the theories are all very different in terms of fundamental ontology or the structure of gravitational interactions? This is the

\footnotetext{
${ }^{2}$ For more on this distinction one can see (Salmon, 1983).

${ }^{3}$ For a fuller philosophical discussion of the confirmation, see (Earman \& Glymour, 1980; Mayo, 1991) and for a more historical look at the expedition see Kennefick (2019).
} 
situation in which contemporary particle physics finds itself and these are the questions of primary interest in this paper. Even with new data from the LHC, it is not obvious which models are being confirmed or disconfirmed by the data. This problem arises from two features: i) models are designed by construction to agree with existing data; ii) they have overlapping predictions for new observations. Thus, even with new empirical data points (such as the discovery of the Higgs boson), one may be unable to distinguish the models, even though they are only transiently underdetermined. What I will show in Sect. 3.2 is that this is precisely the case for the SM Higgs and some BSM Higgs models, like MSSM. This paper sheds some light on the fact that the Higgs observation has been taken to confirm one of these hypotheses more than the others.

The real pay-off of the paper is that this case is not contrived or atypical. The culprit seems to be the agreement among the models' predictions. This only exacerbated by the fact that theory is relatively cheap. Theorists and models builders can produce empirically adequate models with well-worked out predictions far more easily and inexpensively than they can be tested. And so one finds similar conditions at the forefront of many areas of scientific research where there is any number of viable models that make overlapping predictions about new data. Even though these models may propose vastly different mechanisms, causes, ontologies, interactions, and so on, there will be a similar difficulty in establishing which among them has been confirmed if the new data is discovered at these prediction intersections. I will examine the confirmation of the SM Higgs boson, but there are other situations where the same issues for confirmation will arise. In the final section, I propose that eliminative induction is a good way to understand confirmation in these circumstances.

\section{The Case of the SM Higgs}

Before stepping more deeply in the case study, let me briefly describe the models involved. The Higgs mechanism is a central component of the SM that allows for the generation of the masses of elementary particles within a renormalizable gaugetheoretic framework. The mechanism is crucial for the SM because a gauge theory of the electromagnetic and weak forces features both massless and massive particles. The heaviness or short range of the mediating weak particles was known experimentally, but when mass terms are simply added to the Lagrangian by hand, it ruins the renormalizability that is a major motivation for the gauge theory in the first place. In order to generate the masses, the symmetry of the system must be broken spontaneously, rather than explicitly, and this mechanism of spontaneous symmetry breaking was the key insight of Englert and Brout (1964), Higgs (1964), and Guralnik et al. (1964). The proposal is to introduce a complex scalar doublet field to the EW Lagrangian in order to spontaneously break the symmetry and give masses to the intermediate vector bosons and the other SM particles. This field breaks the symmetry due to the 'Mexican hat' shape of its potential. The whole potential is symmetrical, but the lowest points (vacuum states) lie in a circle away from the origin. This means that any particular vacuum configuration of the field has a non-zero value 
called a vacuum expectation value (vev). Because this field always has a value, even in its vacuum state, when particles interact with this field they gain masses proportional to the strength of their interaction with the field.

This one doublet symmetry breaking model has become what is called the SM Higgs and it is the simplest implementation of the Higgs mechanism and is sometimes referred to as the minimal Higgs sector. It is of course possible that the symmetry of the system could be broken by a more complicated Higgs sector, such as by two Higgs doublets, a doublet and a singlet, a triplet, and so on. Models of BSM physics, such as Grand Unified Theories and supersymmetry (SUSY) all involve more complicated Higgs sectors. The question of confirmation is to determine to which of these models the particle discovered at the LHC belongs.

This paper is not the first to treat the Higgs discovery in terms of philosophical confirmation. This has been done for instance in (Dawid, 2017), however, his analysis is of the confirmation of the Higgs mechanism and involves comparing the Standard Model with a Higgs boson to the Standard Model without one. The analysis would not work if one were considering the two particular models with Higgs bosons, like the SM Higgs and minimal supersymmetry, because it relies in part on a No Alternatives Argument. Physicists' confidence in the Higgs mechanism as responsible for particle masses is supported by the fact that are no other good alternative explanations. This argument cannot be used to assess the confirmation of the $S M$ Higgs, because it has many reasonable alternatives. Dawid's analysis focuses on the time prior to empirical support for the hypothesis, i.e., prior to the discovery of a Higgs boson. However, now that a Higgs boson has been discovered, the question of what kind of Higgs it is is the pressing question for many particle physicists. This paper looks at the confirmation process since 2012, when the focus has been on comparing different realisations of the Higgs mechanism.

In order to make this tractable, and since precise predictions of the Higgs mass are basically non-existent, we can allow grouping of mass predictions into reasonably sized bins of $1 \mathrm{GeV}$, as is standard. Second, given that often only upper and lower bounds can be calculated, one could allow that models predict a range of values for the Higgs mass parameter. Thus, instead of a hypothesis $H_{1}$ predicting that the mass of the Higgs $m_{h}$ has a particular value $M_{1}$, it predicts a range of values $m_{h}=\left[M_{\text {low }}, M_{\text {high }}\right]$ that the parameter may take given other constraints in the model. This should allow us to narrow the focus to compare particular realisations of the Higgs mechanism - we can shift from comparing the 'Higgs mechanism' vs. 'Higgs mechanism-alternatives' to 'SM Higgs' vs. 'SM Higgs-alternatives'. To do so, we can specify what these hypotheses are that we should compare. We can state the SM hypothesis about the Higgs in non-technical terms as follows:

The Higgs mechanism is realised by one complex scalar doublet.

This will later be contrasted with a hypothesis that is featured in minimal supersymmetry about an extended Higgs sector, which can be similarly stated:

The Higgs mechanism is realised by two complex scalar doublets.

We can take these to stand in for minimal and extended Higgs sector hypotheses. 


\subsection{Discovery and Confirmation}

Following the initial Higgs discovery announcements, physicists turned their attention to examining not whether there was a Higgs, but what kind of Higgs it was. This would involve either continuing to confirm the SM predictions or finding deviations that might count as evidence in favour of some BSM model. Official reports and summary presentations at major conferences all reported that the particle's observed properties were all within margins of error of the predictions of the SM. This can be seen in (Chall et al., 2018), who present a detailed history of the Higgs discovery and the major physics milestones in the process of its confirmation through such conference summaries and describe the effects of the discovery on the landscape of various BSM models. They note that the discovered particle transitioned from being a "Higgs-like boson" to a "SM-like Higgs boson" as the fact that it was a Higgs become accepted and what remained to be determined was what kind.

There are persuasive reasons for the high confidence that was expressed in the SM Higgs hypothesis. In this section, I review what one may call direct confirmation, or confirmation in isolation from other hypotheses. The most important condition for confirmation is that the predictions of the model match empirical data and I will demonstrate this first. For many philosophers, however, this is not enough. It has been argued that, in addition, in order for a hypothesis to be confirmed by data (i) it should entail a novel fact or (ii) the data should be a good (severe) test of the prediction. I will show that these are also easily satisfied by the SM Higgs hypothesis.

First, as has been summarised by every article and presentation since the discovery, all available data has indicated no significant deviations from SM predictions. ${ }^{4}$ As a strongly indicative example, the SM Higgs boson is predicted to decay into specific particles at precisely determined rates. Detectors do not detect the Higgs boson directly but rather can count the number of times the Higgs decays into a particular final state the detector can pick up. One then compares the number of these decays predicted to the number actually observed, which is called the signal strength $(\mu)$. The closer the number is to 1 , the better is the fit for the SM. In a recent combined analysis, the Particle Data Group has shown that the combined signal strengths across all channels (all final states of Higgs decays) is:

$$
\mu=1.09 \pm 0.04(\exp ) \pm 0.07 \text { (stat) }
$$

and they conclude that "a coherent picture emerges with an excellent consistency between the observation in each channel and the expectation for a SM Higgs boson" (Tanabashi et al., 2018). One could examine all of the reports and studies and combined analyses, but in short, one will find that SM Higgs has been confirmed by its precisely determined predictions.

If one does not consider the accuracy to be sufficient for confirmation, the prediction can also be shown to be novel. The requirement of novelty for confirmation

\footnotetext{
${ }^{4}$ Again, for a more comprehensive philosophical look at the various aspects of the confirmation of the Higgs, one can see (Chall et al., 2018).
} 
stems largely from arguments that data cannot confirm a hypothesis, if the hypothesis was constructed to accommodate the data. Exactly how novelty can be distinguished and how it contributes to confirmation has been much debated in the philosophy of science (Hempel, 1965; Popper, 1959; Worrall, 1978; Zahar, 1973). The motivation for the requirement of novelty is that we are much more inclined to think that a hypothesis gets something right if it makes novel predictions because it precludes the use of ad hoc auxiliaries and speaks to the ability of a hypothesis to truly predict, rather than accommodate after the fact.

One can think about novelty in a few distinct ways. Specifically, it can be distinguished along two orthogonal dimensions. Firstly, it can be either mere temporal novelty or what has been called use novelty. Temporal novelty is when a prediction has been tested in the past and is simply being tested again. E.g. I can make a prediction that tomorrow my pen will drop when I let it go. The particular datum is not yet known and so it should be considered temporally novel and thus confirmatory to at least some small degree as it is a new positive instance. Any time the observation comes later than the prediction, we can say that it is novel in this sense. This is certainly the case with the Higgs as the prediction of the particle was made in 1964 and it was only observed in 2012. Others have held that what matters for confirmation is not whether the prediction came before the observation, but that the evidence is not used in the construction of the hypothesis. This is known as use-novelty and has been the centre of much discussion over the years (for instance, see Giere, 1991; Howson, 1984; Mayo, 1991, 1996; Nunan, 1993; Schurz, 2014; Worrall, 1978, 1989, 2014). The basic idea is that a successful prediction can confirm a theory, but only if that evidence is not used in the creation of the theory, since that would involve counting the evidence twice and lead to a circularity. The temporal aspect is seen as not relevant to the confirmation of the hypothesis. In our case, once again, since the empirical evidence of the SM Higgs only really came about almost 50 years after the proposal, it could not possibly have been used in the construction of the hypothesis, and so it is also novel in the use-novelty sense. One might argue that evidence for the Higgs mechanism started emerging with the discoveries of the $\mathrm{W}$ and $\mathrm{Z}$ bosons, but even this was almost 20 years after the original proposal.

Novelty can also be distinguished by whether it is merely novel by context, or in kind. Novelty in context is similar to temporal novelty, in that it may involve repeating an experiment in a new situation. Astronaut David Scott performed this kind of novel confirmation test when he dropped a feather and a hammer on the moon. Of course, David Scott surprised absolutely no one, and did little to confirm the well-known principle, partly because the test was not novel in kind. Novelty in kind requires something much stronger, such as the famous prediction of general relativity that light would be affected by massive bodies, or in our case that there is a fundamental scalar particle with non-universal couplings that is responsible for breaking the electroweak symmetry. Prior to the discovery, all known scalars (spin0 ) were composite particles, all known fundamental particles were spin-1 (gauge bosons), or spin- $\frac{1}{2}$ (leptons and quarks). Fundamental scalars were not only undiscovered, but thought by many to be problematic, due in large part to the naturalness problem (see Giudice, 2008). 
Some philosophers, such as (Mayo, 1991, 1996), have considered instead that the riskiness of a prediction in an experiment is necessary for its confirmation by that experiment. She argues that what various requirements of novelty are actually aiming for is that the experiment be a a good test (specifically, a severe test) for the hypothesis. ${ }^{5}$ The basic idea is that a hypothesis can only be confirmed by an experiment if it risks being wrong in that experiment, otherwise the positive instance counts for nothing. The riskiness of a hypothesis is not about the probability of its actually being wrong, but the probability of its being found wrong by an experiment if it is in fact wrong. ${ }^{6}$ We can make this more precise by employing Mayo's notion of severe testing. Mayo posits a criterion of severity for confirmation as follows: "Severity Criterion: There is a very high probability that test $T$ would not yield such a passing result, if $h$ is false" (Mayo, 1991, p. 529). She further distinguishes minimally from maximally severe tests. Minimally severe tests occur where the probability of a false hypothesis failing a test is zero, and maximally severe tests where it is one (thus corresponding to a crucial experiment). In between the two extremes one can consider a range of severity. Different probabilities of error detection "provides the basis for distinguishing the well-testedness of two hypotheses-despite their both fitting the data equally well. Two hypotheses may accord with data equally well but nevertheless be tested differently by the data. The data may be a better, more severe, test of one than of the other" (Mayo, 1996, p. 178). The degree of confirmation conferred to a hypothesis is proportional to the severity of the test it passes. The experiments at the LHC plausibly serve as a severe test for the SM hypothesis, because it was highly probable that the test not yield a passing result if it was false. There are many ways in which the SM Higgs hypothesis could have been found to be wrong at the LHC. It would have been incompatible with the SM Higgs if the discovered particle had been charged or had indications of compositeness and in this sense it is a risky hypothesis and it satisfies the severe testing criterion.

Not only was it possible that the SM would be wrong, there were in fact strong expectations leading up to the launch of the LHC that the Higgs sector would demonstrate evidence for BSM physics. The SM is widely believed not to be the end of physics. More complicated Higgs sectors were favoured by the many proponents of supersymmetry, which requires at least an additional Higgs doublet. Some physicists favoured composite Higgs models that would turn electroweak symmetry breaking into just another case of dynamical symmetry breaking and avoid the naturalness problem that accompanies fundamental scalars. ${ }^{7}$ It is worth noting that while there were many risks associated with the prediction of a Higgs boson, i.e. the LHC served as a severe test for the hypothesis, the value of the Higgs mass is a free parameter in the SM. As such, the value of the mass that was measured is not a

\footnotetext{
5 Note that for Popper (1959) this is a sign of corroboration rather than confirmation. The more severe the tests a hypothesis has passed, the more highly it is corroborated. However, for Popper, even very highly corroborated hypotheses do not warrant belief as true as it is only a sign of past performance.

6 Thus, a hypothesis is only risky with respect to an experiment that is testing it.

7 For more on the reasoning behind the SM Higgs and its alternatives, see (Borrelli, 2012; Friederich et al., 2014).
} 
contributing factor in the severity of the test of the hypothesis, as will be elaborated in the following section.

These points make a compelling case that not only was the Higgs mechanism confirmed by the experiments, but so was a particular realisation of this mehcanism, viz. the SM Higgs hypothesis: the predictions of the hypothesis were novel in all senses and it passed a severe test in Mayo's sense. This confirmation by the experiment also translates into high confidence in the hypothesis. Physicists are actively looking for BSM physics, but nevertheless are very confident that the particle is a SM-like Higgs boson. In practice, physicists mostly assume that the particle is exactly SM-like and incorporate that into the background signal in searches for new physics. The following section introduces a concern for this high level of confidence, which is that in a comparison, the SM Higgs appears less strongly confirmed by the discovery than a rival hypothesis. This follows primarily from the fact that the value of the Higgs mass in the SM is a free parameter and not a genuine prediction. While the SM hypothesis about the Higgs did risk being wrong at the LHC, other hypotheses could easily be riskier by providing, for example, constraints on the mass. What we will see is that another hypothesis in fact passed a more severe test of the same data. This is a point made by (Chall et al., 2018), which I take as a jumping-off point for this discussion of confirmation: "MSSM made a bolder, riskier prediction by providing more precise theoretical bounds on the Higgs mass, and so the evidence ought to more strongly confirm MSSM" (p. 19). The concern will be resolved in the final section, where a more appropriate modelling framework is introduced.

\subsection{A Comparison with Minimal Supersymmetry}

Models that predicted additional low energy particles or large deviations from the SM were increasingly ruled out as LHC data was analysed. BSM models were being 'squeezed out' of their remaining parameter spaces, leaving the SM standing mostly alone. The exceptions to this are models that could accommodate a SM-like Higgs boson, like minimal supersymmetry (MSSM). Despite the fact that MSSM predicts many additional particles that have not been observed, and hence the model as a whole in under significant pressure, the model's hypothesis about the Higgs was spot on. MSSM is a popular BSM model and was heavily favoured prior to the LHC, because, as a supersymmetric model, it offers solutions to many perceived problems of the SM and it features the simplest Higgs sector beyond the SM, the two Higgs doublet model, or 2HDM. As the name suggests, the basic structure of this Higgs sector is that in addition to the one Higgs doublet of the SM, there is another one. For one doublet, there is a single physical boson remaining after the degrees of freedom are 'eaten' by the $\mathrm{W}^{ \pm}$and $\mathrm{Z}$ bosons. For two doublets, there are five physical Higgs bosons remaining. There are two charged Higgses $H^{ \pm}$, a pseudo-scalar $A$, and two CP-even neutral scalars $h$ and $H$.

Most importantly for our discussion, MSSM necessarily involves an allowable mass range for the Higgs, such that if the particle had been too heavy, the model would have been discounted. Of particular interest is the value of mass of the 
lightest Higgs boson, $m_{h}$, which could be identified with the boson discovered at the LHC. At lowest order, the lightest Higgs mass is constrained by other mass values in the following way:

$$
m_{h}^{2}=\frac{1}{2}\left(\left(m_{A}^{2}+m_{Z}^{2}\right) \pm \sqrt{\left(m_{A}^{2}-m_{Z}^{2}\right)^{2}+4 m_{A}^{2} m_{Z}^{2} \sin ^{2} 2 \beta}\right),
$$

as shown in the Higgs Hunter's Guide (Gunion et al., 2000). The tree-level mass is strongly bounded from above by the mass of the Z boson, where $m_{h}=m_{Z}|\cos 2 \beta|$ and the mass of the $\mathrm{Z}$ has been experimentally determined to be about $91 \mathrm{GeV}$. What this means is that large radiative corrections are needed to increase the bound to reach the observed Higgs mass of $125 \mathrm{GeV}$. This can be accomplished with minimal supersymmetry, but it cannot be pushed much higher. On minimal supersymmetric models, there is an upper bound on $m_{h}$ at around $130 \mathrm{GeV}$. Thus, the experimentally determined values of the model's parameters leaves open a narrow window for the possible mass value of the Higgs.

On the contrary, the SM predicts that there should be a fundamental scalar particle, but it famously does not predict its mass. Theoretically, the SM has no preference for the value of the Higgs mass - it is a free parameter and its value is not a prediction. From the SM Higgs Lagrangian, we can get the following mass terms at tree level for the gauge bosons:

$$
m_{W^{ \pm}}^{2}=\frac{v^{2}}{4} g^{2}, m_{Z}^{2}=\frac{v^{2}}{4}\left(g^{2}+g^{\prime 2}\right), m_{\gamma}=0,
$$

and the Higgs mass is given by

$$
m_{h}=\sqrt{\frac{\lambda}{2}} v
$$

where $v$ is the Higgs vacuum expectation value, $\lambda$ is the Higgs self-coupling, and $g$ and $g^{\prime}$ are coupling constants. Thus, unlike the masses of the the photon, $\mathrm{W}^{ \pm}$, and $\mathrm{Z}^{0}$, the Higgs mass on the SM is a free parameter that depends only on its coupling to itself and not on any other SM physics. Unlike in the case of the MSSM Higgs, the experimentally measured values of the masses of the $\mathrm{W}$ and $\mathrm{Z}$ bosons is compatible with any value of the SM Higgs mass. The other properties of the SM Higgs can all be precisely calculated as a function of its mass, but the mass must be experimentally determined.

However, the mass of the Higgs in the SM cannot be just anything; there are broad theoretical considerations that place some upper and lower bounds on the Higgs mass and these constraints depend on the scale of new physics. There are upper bounds to preserve unitarity and lower bounds to prevent triviality. If the Higgs mass is too high, perturbation theory breaks down and unitarity is violated. This occurs when $m_{h} \gtrsim 800 \mathrm{GeV}$ (Dawson \& Willenbrock, 1989; Hasenfratz, 1989). On the other hand, because of the strong dependence on the top quark mass, if the Higgs mass is too low, where $m_{h} \lesssim 70 \mathrm{GeV}$, then there will be vacuum instability 
(Casas et al., 1996; Elias-Miro et al., 2012). ${ }^{8}$ Additionally, by the time the LHC started up, ATLAS and CMS could take into account existing exclusions from LEP and Tevatron searches. The Higgs was experimentally excluded at masses less than 114.4 GeV and between about 159 and $180 \mathrm{GeV}$ (De Roeck \& Jenni, 2011). Experimentally, there was smaller window within which one could reasonably expect to find a SM Higgs boson, but these latter constraints on the mass do not follow from the model-it is not a genuine prediction about the value of the mass. If one wants to assess the probability of a Higgs being in a certain mass range given confidence in its existence and the previous searches, then it is quite reasonable to include nothing but previous exclusion ranges. In this case there is no comparing the different models and one would be analysing the probability of finding $a$ Higgs, more along the lines of the lines of the argument given in (Dawid, 2017). However, in order to compare the models one must look at the models' predictions. The models are compatible with different ranges of the mass value and so the same experiment will provide a test with different severities. Experimental constraints that are only the results of not already having found the Higgs at previous experiments do not reflect the precision of the hypotheses and so will not be a factor in the severity of the tests or in determining how strongly the data confirms the hypotheses. ${ }^{9}$ Thus, if we are to be generous and admit that the SM makes a prediction for the Higgs mass at all, it should be no narrower than $70-800 \mathrm{GeV}$.

At the end of Sect. 3, I introduced the hypotheses that we were to compare-that there is either one or two doublets involved in the Higgs mechanism. Now we are at a point where we can compare the hypotheses in observational rather than theoretical terms by specifying the properties of the Higgs boson that could be detected at the LHC, most importantly, the allowable mass range. We can then formulate the predictions for the Higgs including allowable mass ranges as follows:

SM Higgs hypothesis: There is a SM-like Higgs boson with $m_{h} \epsilon[70,800] \mathrm{GeV}$

MSSM Higgs hypothesis: There is a SM-like Higgs boson with $m_{h} \epsilon[70,130]$ $\mathrm{GeV}$

The Higgs boson was discovered to have the mass of $125 \mathrm{GeV}$ and so the two models have overlapping predictions at the mass where the Higgs boson was found to be. This problem is not unique to these two models, but in fact is common to any viable model in the Higgs sector that can accommodate a SM-like Higgs boson. To state the problem more precisely, any model that remains viable after the discovery also 'predicts' a SM-like Higgs boson. This is a phenomenon known as SM alignment. In MSSM, SM alignment can be naturally achieved if the masses of the four heavier Higgses is far greater than the light one discovered. As the mass of the next lightest Higgs increases, the lightest Higgs approaches being SM-like. If the masses are sufficiently decoupled, then the lightest Higgs becomes exactly SM-like, since one

\footnotetext{
8 This assumes that there is no new physics below $1 \mathrm{TeV}$.

9 This is distinct from considering the other empirical constraints, such as the measured values $\mathrm{W}$ and $\mathrm{Z}$ masses, the determination of which are necessary for the predictions of the Higgs mass.
} 
can integrate out the heavy fields and recover an effective one-Higgs doublet (Haber et al., 2017; Heinemeyer et al., 2012). This is known as the Higgs decoupling limit.

Given the lack of results in searching for light heavy Higgses, it seems that if there are heavy Higgses, they will be in the decoupling limit. The only way to distinguish models that are SM-aligned would be to confirm predictions elsewhere in the model, such as finding a new particle. However, the decoupling in the Higgs sector could mean that evidence of a non-minimal sector is out of reach. In a recent paper analysing MSSM parameters, Heinemeyer et al. (2018) came to the following conclusion about the prospects of ruling out heavy Higgses:

The SUSY Higgs boson mass scale is found above $\sim 1.3 \mathrm{TeV}$, rendering the light MSSM Higgs boson SM-like, in perfect agreement with the experimental data...Consequently, the reduced MSSM is in natural agreement with all LHC measurements and searches. The SUSY and heavy Higgs particles will likely escape the detection at the LHC, as well as at ILC and CLIC.

(Heinemeyer et al., 2018)

Data indicates that if there is an extended Higgs sector, then the heavy Higgses will be too heavy to detect and while physicists are precisely probing the SM for deviations, it may be that conclusive support for a minimal Higgs will not be forthcoming, even at the next generation of detectors.

LHC data has put constraints on MSSM parameters and has pushed the mass values of additional particles beyond the reach of the LHC (otherwise their effects would be detected). But this implies that its achievable predictions are indistinguishable from those of the SM. The only difference is the range of allowed values for the Higgs mass on both models; the precision of their theoretical predictions. Let me present a short formal Bayesian argument that, counter-intuitively, the Higgs discovery more strongly confirms the MSSM than the SM. ${ }^{10}$ Bayes' theorem is written as:

$$
p(H \mid D)=\frac{p(D \mid H) p(H)}{p(D)}
$$

where $p(H \mid D)$ is the conditional posterior probability for $H$ given data $D, p(D \mid H)$ is called the likelihood and is the probability of finding the data $D$, given the hypothesis $H, p(H)$ is the prior degree of belief in the hypothesis, and $p(D)$ is the expectedness or prior of the data that also serves to normalise the probability. ${ }^{11}$ One can also say that the posteriors are proportional to the likelihood times the prior,

$$
P(H \mid D) \propto p(D \mid H) p(H),
$$

and we can focus on these two values to assess the posteriors. We want to assess the ratio of posteriors of the two hypothesis,

\footnotetext{
${ }^{10}$ It is important to keep in mind that this is confirmation in the sense of increase in firmness by this experiment and not a measure of overall support for the models. Of course, there has not been any significant evidence indicating any of the SUSY particles predicted by MSSM and many low energy SUSY models are under significant pressure.

11 The equation is often presented as conditional on background knowledge $K$.
} 


$$
\frac{p\left(H_{0} \mid D\right)}{p\left(H_{1} \mid D\right)},
$$

where $H_{0}$ is the SM hypothesis and $H_{1}$ is the MSSM. In order to compare the posteriors we need to establish the likelihoods and the priors for both hypotheses (from 6). These models do not predict particular values for the mass, but rather are equally compatible with values within a range; it makes no difference to the model if the measured value is towards the high end of the range or in the middle. Because there is no theoretical preference for the masses within the allowed ranges, we should invoke a principle of indifference and take a flat likelihood over the allowed range. Thus, the probability will be given by $p\left(D \mid H_{0}\right)=k_{0}$, where $k_{0}$ is a constant given by 1 divided by the range of possible mass values. This represents the likelihood of finding the particle in any given $1 \mathrm{GeV}$ bin in the hypothesis' range. Given the earlier stated ranges, we can give the likelihoods as follows:

$$
k_{0} \approx 1 / 730
$$

for the SM and

$$
k_{1} \approx 1 / 60
$$

for MSSM. This states that if the prediction of the model is correct, there is an equal likelihood of measuring the mass at any point in the allowed range. We can compare the relative strength of confirmation of $D$ on $H_{0}$ and $H_{1}$ by using the following ratio:

$$
\begin{aligned}
& \frac{p\left(H_{0} \mid D\right)}{p\left(H_{1} \mid D\right)}=\frac{k_{0} p\left(H_{0}\right)}{k_{1} p\left(H_{1}\right)} \\
& \frac{p\left(H_{0} \mid D\right)}{p\left(H_{1} \mid D\right)}=\frac{60 p\left(H_{0}\right)}{730 p\left(H_{1}\right)}
\end{aligned}
$$

At this point, the argument should be clear on the basis of the likelihood ratio which hypothesis is more strongly confirmed.

One can now move on to estimate the priors. The prior for the SM should be quite high as the model is the simplest implementation of the Higgs mechanism and many other SM predictions about the $\mathrm{W}$ and $\mathrm{Z}$ bosons have been precisely confirmed. However, physicists also had very high expectations for new physics and so the MSSM hypothesis, as the simplest and most highly-favoured of the BSM models of electroweak symmetry breaking, should also be quite high. Instead of making a single, and rather arbitrary, quantitative estimate, let me say the following instead: given the likelihoods established above, the priors would have to differ substantially in order for them to give a different result. Given the ratios in 11, in order for the SM to have been more strongly confirmed, the SM prior $p\left(H_{0}\right)$ would have to be more than $12 \times$ higher than for MSSM to make up for the difference in the ranges. Unless an argument for such a difference can be convincingly made, this gives us the following result: the posterior for the MSSM is greater than that for the SM: 


$$
p\left(H_{0} \mid D\right)<p\left(H_{1} \mid D\right) .
$$

Because every other distinction between the model is held fixed, the only differentiating factor is the range of the prediction for the mass value. Thus, we have a result that ceteris paribus the riskier successful prediction provides a stronger confirmation and ceteris really is paribus due to SM-alignment (all other properties of the (lightest) Higgs are expected to be the same on both models).

Given the experimentally determined values of other parameters, the SM made no prediction about the value of the Higgs mass while MSSM did. The masses of the W and $\mathrm{Z}$ bosons place constraints on possible values of the MSSM Higgs mass, but not on that of the SM Higgs mass. But, if one is to be generous and allow that both models made a prediction, the SM made a much broader prediction than the MSSM and thus passed a less severe test and was less strongly confirmed by this measurement. Not only did MSSM predict all the same properties of the boson as the SM (and was thus equally confirmed on all those fronts), it predicted that its mass would lie within a much smaller range. Given existing empirical constraints, such as the value of the Z mass, MSSM made a riskier prediction by allowing for a narrower range of possible values for the Higgs mass. Because the hypothesis passed a more severe test, the evidence ought to confirm its hypothesis even more strongly. ${ }^{12}$

There should be no doubt that the SM Higgs was highly confirmed by the LHC data, but many other models were as well—any model that could accommodate a SM-like Higgs. What I take from this is the general lesson that what is selected, or favoured, by experiment is a class of models (or theories or hypotheses) that can accommodate the experimental results. Whenever a measurement is made, every hypothesis that can accommodate that measurement is increasingly confirmed, but not all to the same degree. Interestingly, this seems to be the case for incompatible models. If two models $M_{1}$ and $M_{2}$ have hypotheses $H_{1}$ and $H_{2}$ respectively that are compatible with some data $D_{1}$, then both models will be confirmed by the data. This should be the case even if the models have other hypotheses that counterpredict each other, i.e. if the truth of the hypotheses of one model entails the falsehood of the hypotheses of the other. In our case, these counterpredictions would be about other Higgses. Both this fact and the putatively stronger confirmation of the SM over MSSM will be discussed in the following section.

\section{A Resolution}

\subsection{Two Puzzling Features}

The analysis presented so far reveals two features that request explanation: (1) the fact that the SM is taken to be so much more strongly confirmed by the data than the MSSM even though according to the analysis they should at best have received the

\footnotetext{
${ }^{12}$ Note that this is not the same as to say that, all things considered, MSSM is more strongly supported by the data than the SM.
} 
same confirmation; (2) the fact that these two models were confirmed by the data even though they are counterpredictive. Neither of these is necessarily problematic, but they seem curious at first glance, and so I will discuss them in turn in order to better understand the situation.

Given the analysis presented above, one might expect that either the MSSM hypothesis would be taken by physicists to have been more strongly confirmed than the SM hypothesis or to have been confirmed just as strongly. This is not what one finds. One finds that the Higgs discovery is everywhere touted as a victory for the SM. Why is that? One reason may be that there is a conflation between confirmation as firmness and increase in firmness, as mentioned on p. 3. What this analysis does not intend to show is that MSSM is more strongly empirically supported (firmness). The point is rather that the experiments that discovered the Higgs proved to be a more severe test of the MSSM Higgs than the SM Higgs and hence plausibly conferred a higher degree of confirmation. The major difference in terms of confirmation as firmness is of course that there is yet no indication of any supersymmetric partners. Further, the LHC constraints that decouple the additional new physics also mean that various MSSM parameters require fine-tuning, which makes it less attractive to many physicists. There is also the contingent historical fact that the SM hypothesis was developed first. It is also simpler than BSM hypotheses that involve extended Higgs sectors and other new physics besides. Without strong evidence in favour of additional new physics in the Higgs sector, the SM Higgs may be seen to stand as the strongest supported by evidence. Its simplicity and relative priority may mean that the SM Higgs hypothesis could have taken on a kind of default position, which like a null hypothesis, may stand until it is rejected in favour of an alternative, irrespective of whether an alternative would have performed just as well under the same tests.

Further, Bayesian confirmation theory provides many tools for updating probabilities, but there is flexibility in how one sets up a model of confirmation. It is possible that some factors, including those I have mentioned, may be given significant weighting which would change one's assessments of the relative priors of the two hypotheses. One factor in particular seems most relevant here: the expectation for new physics. Due to its central role in particle physics (the Yukawa couplings to quarks and leptons), the Higgs boson is a powerful tool, or perhaps even laboratory, for the investigation of possible new physics and many physicists had (and still have) high expectations for the sector to reveal evidence of BSM physics. Different expectations could have led to the assignment of very different priors. ${ }^{13}$

The riskiness of the hypotheses was what made the biggest difference to their relative confirmation. Because any new physics would be sufficiently heavy enough to be decoupled, it can be integrated out, and in the end one is essentially comparing the one Higgs doublet of the SM with a free mass parameter to an effective one Higgs doublet of MSSM with only a tightly allowed mass range. The difference in the allowable mass ranges implies that the hypotheses passed tests of very different severity in the same experiment. In order to rebuff the results of the analysis, one

13 It is important to keep in mind that these priors are on the hypotheses as stated above (Sect. 3.2).
It would be very difficult, if not impossible, to assess priors or credences in the SM itself, given that
no physicist thinks that it is the final theory of physics, and as such the SM + no BSM physics should 
could deny that the degree of riskiness (the precision of the prediction) is indicative of the degree of confirmation conferred to the model by the test. One could still take riskiness to be necessary, in the sense that the experiment could have possibly shown that the hypothesis was false (both for the SM and MSSM Higgs hypotheses), but deny that passing a more severe test confers a stronger degree of confirmation. That is, one could reject the distinctions that the severe testing approach makes and that I have sketched in the Bayesian model. But it is very plausible that more severe tests confer a higher degree of confirmation, and luckily one need not give it up. A likely alternative diagnosis is that there is something overly simplistic in the way the confirmation comparison was modelled as the likelihood ratio of two models in 3.2. In the following subsection, I will outline a model that better captures how the confirmation process took place, viz. eliminative inductivism (EI). EI is naturally suggested to me by the final major reason for the confirmation discrepancy, which is that the SM Higgs hypothesis is being confirmed not only because its predictions are in accordance with the data, but because the space of alternative models is being reduced.

\subsection{Eliminative Induction}

Eliminative Inductivism (EI) has been described as a kind of inverted Popperianism, where corroboration of a hypothesis comes from successful attempts to falsify its alternatives. In our case, the idea is that the SM hypothesis is being confirmed as BSM models with predictions within the sensitivity of the LHC are being pushed to the edges of their parameter spaces. In other words, the SM is being confirmed because its alternatives are being disconfirmed. This is the reasoning of eliminative inductivism. As a toy example, if there are ten equally possible murder suspects and eight of them have been eliminated with strong alibis, then even without direct evidence in favour of the either of the remaining two, probability assessments about the remaining suspects should increase. Interestingly, this is the case even though the truth of one of the remaining hypotheses (person A did it) entails the falsehood of the other (person B did it), assuming they were not in cahoots.

John Earman (1992) made a 'plea' for eliminative inductivism as a way of making good use of the Bayesian framework. Earman looks at the almost explicit use of EI in gravity theories and I wish to present BSM models, in particular in the Higgs sector in light of the discovery, as another excellent example. Though Earman notes that EI falls outside of Bayesian orthodoxy, this has been challenged by (Hawthorne, 1993) who claims that Bayesianism can be understood as a bookkeeping device for the elimination process. I want to make no claims about the relation between EI and

Footnote 13 (continued)

receive a prior of 0 . Perhaps one should not understand this probability assessment as to the literal truth of the SM. What seems to have increased is belief in a higher range of validity. In a sense, the SM is increasingly confirmed as the energy range up to which it is valid increases. This is not the case with the SM Higgs hypothesis, which could be reasonably thought to be true, in the sense that there might actually be one Higgs doublet. 
Bayesian orthodoxy nor between EI and the way science ought to proceed. Rather, the I think that EI can provide insight into the confirmation process following the Higgs discovery and many other cases under similar conditions.

The key to making a case for increasing confirmation by eliminative induction is to establish that the alternative hypotheses are being disconfirmed. This could be difficult to rigorously establish for a few reasons. Though I will mention some of these, I think they can be accepted as modelling idealisations and one can proceed with the EI. The first is that the space of possibilities, of all the hypotheses, needs to be known and partitioned. If the space of possible hypotheses includes a catch-all hypothesis standing in for a infinite conjunction of hypotheses that have not yet been conceived, then there is no way to assign a probability to that hypothesis and no way to update the probabilities by Bayes' Theorem.

EI can reasonably be applied in the Higgs case because the space of possibilities (of alternative models) is reasonably well mapped out. It is in fact very well mapped out if one is considering the space of hypotheses that are within the sensitivity of the LHC. Trying to model the confirmation of the SM hypothesis $(H)$ as against SM alternatives $(\neg H)$ is impossible unless one artificially delimits the alternative possibilities to those that have been well mapped out and are accessible at LHC energies. Doing this can then give us an idea of how the probability among conceived alternatives (its realistic rivals) has been affected by the data. ${ }^{14}$

The second is that alternatives are never really eliminated. The situation would be more certain if one could proceed deductively by completely eliminating hypotheses and redistributing the probabilities among the fewer alternatives. However, in practice with real scientific hypotheses, this will not be the case. This is something that is addressed in (Hawthorne, 1993), where deductive and inductive versions of EI are distinguished. Even in cases where the probabilities cannot be pushed exactly to 0 or 1 , it can be shown with various convergence theorems that the posterior for the true hypothesis will approach 1 as those for false hypotheses approach $0 .{ }^{15}$ Even in the absence of rigorous Martingale convergence theorems, the amount and precision of the data allow us to very strongly confirm and disconfirm hypotheses and even estimate the confidence with which this is done. This is standard practice in particle physics. If there is 5 sigma confidence ${ }^{16}$ that the mass of the Higgs is within a certain interval, then hypotheses that predict a Higgs outside of that range are strongly disconfirmed and no longer worthy of serious consideration. At the same time, hypotheses that accord with the observed mass are increasingly confirmed by the updating of probabilities given in Eq. 13 below.

Though certain conditions need to be met for EI, they are none other than those required to make a standard rigorous Bayesian argument proceed. In Earman's own words, "in the absence of the conditions needed to make sophisticated eliminative

\footnotetext{
14 If one was concerned with the strict truth of the hypothesis, then one cannot ignore any of its alternatives. This leads to many issues which are discussed in (Stanford, 2006).

15 See (Hawthorne, 1993) for details on the theorems and their assumptions and applicability.

16 This corresponds to estimating that the chance of the observation being a statistical fluke is 1 in 3.5 million.
} 
induction work, Bayesianism offers no satisfying account of the probabilifications of scientific theories" Earman (1992, p. 167).

EI requires no special mathematical machinery. Bayes' Theorem can be written in the following way so as to make the elimination process explicit:

$$
p\left(H_{k} \mid \bigwedge_{i \leq n} D_{i}\right)=\frac{p\left(H_{k}\right) \times p\left(\bigwedge_{i \leq n} D_{i} \mid H_{k}\right)}{\sum_{j=1}^{N} p\left(\bigwedge_{i \leq n} D_{i} \mid H_{j}\right) \times p\left(H_{j}\right)}
$$

This equation tells us what the probability of a particular hypothesis $H_{k}$ is given the evidence, where $\bigwedge_{i \leq n} D_{i}$ represents the totality, the conjunction, of the evidence $D_{i}$ collected up to that point $n$. In the numerator, we have the recognizable prior times likelihood and in the denominator we have the sum of the likelihood times the prior for each of $N$ hypotheses. Whenever the probability of a hypothesis, say $H_{l}$, approaches zero, its contribution to the sum of the denominator approaches 0 . Whatever probability it had is redistributed over the remaining hypotheses, because the sum of the probabilities of the remaining hypotheses must still add to 1. Again, in reality, the probability may only go so low as to make it unworthy of consideration and effectively eliminated. Nonetheless, this shows how the decreasing confirmation directly entails the increasing probability of others under certain conditions. Given some evidence $D_{k}$, any number of hypotheses could be effectively eliminated and likewise confirmed. In our case, the value of the Higgs mass was incompatible with some models, but compatible with others, including SM and MSSM. Thus regardless of the fact that the models have many hypotheses that counterpredict each other (where the truth of $H_{1}$ entails the falsehood of $H_{2}$ ) they could both be confirmed by the data.

The models that were disconfirmed were those that could not accommodate a Higgs with the observed mass, predicted non-SM like properties of the Higgs, such as composite Higgs models, and models that featured no Higgses, so-called Higgsless models. A strong degree of confirmation was then conferred on the remaining models. This is a picture that only emerges when one considers the effect on the rival hypotheses, and hence the importance of thinking about confirmation in the EI framework. The hypotheses that were confirmed are the ones that are compatible with the discovery of the SM-like Higgs and the values of all the measured properties. This includes both the SM Higgs, and the MSSM Higgs, and many others besides. In fact, it includes any model that recovers a SM-like Higgs, as far as those properties have been determined. ${ }^{17}$ Many of these surviving models posit additional particle content that counter-predict each other; they differ with respect to the particles they predict and their properties. And yet a whole class of these models has been confirmed by the LHC data and not all to the same degree. After a second glance, this is not surprising, and what I am suggesting is that the the best way to understand how the confirmation and disconfirmation of various Higgs hypotheses took place is as an eliminative induction.

\footnotetext{
17 There are a huge number of distinct predictions for the mass range of the Higgs and it is not possible to describe them here. To get a sense of how impossible this would be, see (Dissertori, 2015), who pre-
} 


\subsection{Conjectures and Disconfirmations}

I take this study to be indicative, not only of a way of understanding how Higgs hypotheses have been confirmed, but of the way confirmation proceeds in many areas of scientific research. Many methodologies of science have focused on the positive development of a research program, where scientists try to confirm and complete a theory, attempting to test new predictions, resolve existing anomalies and the like. What seems to be at work across the field of particle physics is a kind of negative development, wherein scientists are trying to find deviations from an accepted theory in order to guide the way towards a new theory. The Higgs boson was the final missing element in the SM and in some ways marked its completion. But the SM is not completely complete: many of its predictions have yet to be tested, such as the Higgs self-coupling, and with more precision, room would remain to discover deviations where there currently are none. The SM is also not final: it does not account for neutrino masses or strong CP violation, offers no dark matter candidate, and makes no mention of gravity, among other things. In short, physicists are busy constructing alternatives to, or extensions of, the SM and are in need of data to adjudicate between them.

I do not want to claim that this is the way that all science operates, but I find it reasonable that it would proceed similarly in cases where there a number of overlapping predictions, an abundance of theory, and relatively little data. There, one would find a multitude of empirically adequate models waiting to confront new data, and hopefully with distinct achievable predictions. When some new data is gathered, some subset of the models may not be compatible with the data and would be disconfirmed. It is worth stressing that this is not disconfirmation in the sense of being ruled out or refuted, but merely made less likely in the Bayesian sense, potentially to the point of no longer being seriously considered. But some other subset of models may be able to accommodate this new data and would correspondingly be increasingly confirmed in just the way that the EI shows. Confirmation may be a matter of just surviving. To borrow an out-of-context passage from van Fraassen (1980, p. 40): "any scientific theory is born into a life of fierce competition, a jungle red in tooth and claw. Only the successful theories survive..."

With so many precise, empirically adequate models on the market, it becomes even clearer that the time of belief in crucial experiments is over. It may once have been demonstrable by imagining an in-principle competitor theory $T^{\prime}$, but now these have taken the form of actual models with well-worked out predictions. These models are empirically adequate, but are not empirically indistinguishable; they merely overlap with some degree of confidence on the values of some achievable (and soon achievable) observations. What one finds when looking at confirmation is that is not one model that is confirmed by data, but whole classes of models. This presents some difficulties when it comes to modelling the confirmation of these models. The

Footnote 17 (continued)

sents a list of 125 distinct predictions for the Higgs mass, with upper and lower bounds, and the other particles entailed by those models. 
investigation was spurred on by the seemingly disproportionate amount to which the SM Higgs hypothesis is taken to have been confirmed, given that it made no predictions about the mass of the Higgs boson. The fact of SM-alignment and Higgs decoupling limit make it difficult to disentangle the various hypotheses about the Higgs and their respective confirmations. This paper has looked at one instance where classes of models have been confirmed and presented a promising way of understanding how this confirmation takes place.

Acknowledgements I would like to thank the members of my research unit, Peter Mättig and Michael Stöltzner, for their support, feedback, and input on many versions of the paper. I would also like to thank Radin Dardashti and Niels Martens for their comments on early drafts, as well as two anonymous reviewers for their helpful comments and suggestions.

Funding Open Access funding enabled and organized by Projekt DEAL. Funding was provided by DFG (Grant No. FOR 2063).

Open Access This article is licensed under a Creative Commons Attribution 4.0 International License, which permits use, sharing, adaptation, distribution and reproduction in any medium or format, as long as you give appropriate credit to the original author(s) and the source, provide a link to the Creative Commons licence, and indicate if changes were made. The images or other third party material in this article are included in the article's Creative Commons licence, unless indicated otherwise in a credit line to the material. If material is not included in the article's Creative Commons licence and your intended use is not permitted by statutory regulation or exceeds the permitted use, you will need to obtain permission directly from the copyright holder. To view a copy of this licence, visit http://creativecommons.org/licen ses/by/4.0/.

\section{References}

Borrelli, A. (2012). The case of the composite Higgs: The model as a "Rosetta stone" in contemporary high-energy physics. Studies in History and Philosophy of Modern Physics, 43(3), 195-214.

Carnap, R. (1962). Logical foundations of probability (2nd ed.). University of Chicago Press (62052505).

Casas, J., Espinosa, J., \& Quirós, M. (1996). Standard model stability bounds for new physics within LHC reach. Physics Letters B, 382(4), 374-382.

Chall, C., King, M., Mättig, P., \& Stöltzner, M. (2018). From a boson to the standard model Higgs: A case study in confirmation and model dynamics. Synthese, 198, 1-29.

Dawid, R. (2017). Bayesian perspectives on the discovery of the Higgs particle. Synthese, 194, 377-394.

Dawid, R., Hartmann, S., \& Sprenger, J. (2015). The no alternatives argument. The British Journal for the Philosophy of Science, 66(1), 213-234.

Dawson, S., \& Willenbrock, S. (1989). Unitarity constraints on heavy Higgs bosons. Physical Review Letters, 62, 1232-1235.

Dissertori, G. (2015). The pre-LHC Higgs hunt. Phil. Trans. Roy. Soc. Lond. A, 373(2032), 1-12. https:// arxiv.org/abs/0708.3344.

De Roeck, A., \& Jenni, P. (2011). Discovery physics from atlas and CMS at the LHC. Proceedings of Science, 338, 1-21.

Earman, J. (1992). Bayes or bust? A critical examination of Bayesian confirmation theory. MIT Press.

Earman, J., \& Glymour, C. (1980). Relativity and eclipses: The British eclipse expeditions of 1919 and their predecessors. Historical Studies in the Physical Sciences, 11(1), 49-85.

Elias-Miro, J., Espinosa, J. R., Giudice, G. F., Isidori, G., Riotto, A., \& Strumia, A. (2012). Higgs mass implications on the stability of the electroweak vacuum. Physics Letters B, 709, 222-228.

Englert, F., \& Brout, R. (1964). Broken symmetry and the mass of gauge vector mesons. Physical Review Letters, 13(9), 321-323. 
Friederich, S., Harlander, R., \& Karaca, K. (2014). Philosophical perspectives on ad hoc hypotheses and the Higgs mechanism. Synthese, 191(16), 3897-3917.

Giere, R. N. (1991). understanding scientific reasoning (3rd ed.). Holt, Rinehart, and Winston.

Giudice, G. F. (2008). Naturally speaking: The naturalness criterion and physics at the LHC. World Scientific. (pp.155-178)

Gunion, J. F., Haber, H. E., Kane, G. L., \& Dawson, S. (2000). The Higgs hunter's guide. Frontiers in Physics, 80, 1-404.

Guralnik, G., Hagen, C. R., \& Kibble, T. W. (1964). Global conservation laws and massless particles. Physical Review Letters, 13(20), 585-587.

Haber, H., Heinemeyer, S., \& Stefaniak, T. (2017) The impact of two-loop effects on the scenario of MSSM Higgs alignment without decoupling. Eur. Phys. J. C 77(742), 742-762. https://arxiv.org/ abs/1401.0152.

Hasenfratz, P. (1989). Upper bound on the Higgs meson mass. Nuclear Physics B-Proceedings Supplements, 9, 3-17.

Hawthorne, J. (1993). Bayesian induction is eliminative reasoning. Philosophical Topics, 21(1), 99-138.

Heinemeyer, S., Mondragón, M., Tracas, N., \& Zoupanos, G. (2018). Reduction of the parameters in MSSM. Journal of High Energy Physics, 2018(8), 150.

Heinemeyer, S., Stal, O., \& Weiglein, G. (2012). Interpreting the LHC Higgs search results in the MSSM. Physics Letters B, 710(1), 201-206.

Hempel, C. G. (1965). Studies in the logic of confirmation. Free Press.

Higgs, P. (1964). Broken symmetries and the masses of gauge bosons. Physical Review Letters, 13(16), 508.

Howson, C. (1984). Bayesianism and support by novel facts. British Journal for the Philosophy of Science, 35(3), 245-51.

Kennefick, D. (2019). No shadow of a doubt. Princeton Press.

Mayo, D. G. (1991). Novel evidence and severe tests. Philosophy of Science, 58(4), 523-552.

Mayo, D. G. (1996). Error and the growth of experimental knowledge. Chicago Univiersity Press.

Nunan, R. (1993). Heuristic novelty and the asymmetry problem in Bayesian confirmation theory. The British Journal for the Philosophy of Science, 44(1), 17-36.

Popper, K. R. (1959). The logic of scientific discovery. Routledge.

Salmon, W. C. (1983). Confirmation and relevance. In P. Achinstein (Ed.), The concept of evidence. Oxford University Press.

Schurz, G. (2014). Bayesian pseudo-confirmation, use-novelty, and genuine confirmation. Studies in History and Philosophy of Science Part A, 45(1), 87-96.

Stanford, P. K. (2006). Exceeding our grasp: Science, history, and the problem of unconceived alternatives. Oxford University Press.

Tanabashi, M., Hagiwara, K., Hikasa, K., Nakamura, K., Sumino, Y., Takahashi, F., et al. (2018). Review of particle physics. Physical Review D, 98, 030001.

van Fraassen, B. C. (1980). The scientific image. Clarendon library of logic and philosophy. Clarendon Press.

Worrall, J. (1978). The ways in which the methodology of scientific research programmes improves on Popper's methodology. In G. Radnitzky \& G. Andersson (Eds.), Progress and rationality in science (pp. 45-70). Springer.

Worrall, J. (1989). Structural realism: The best of both worlds? Dialectica, 43(1-2), 99-124.

Worrall, J. (2014). Prediction and accommodation revisited. Studies in History and Philosophy of Science Part A, 45, 54-61.

Zahar, E. G. (1973). Why did Einstein's program supercede Lorentzs'? British Journal for the Philosophy of Science, 24, 95-262.

Publisher's Note Springer Nature remains neutral with regard to jurisdictional claims in published maps and institutional affiliations. 